Evaluation of friction enhancement through soft polymer micro-patterns in active capsule endoscopy

This article has been downloaded from IOPscience. Please scroll down to see the full text article.

2010 Meas. Sci. Technol. 21105802

(http://iopscience.iop.org/0957-0233/21/10/105802)

View the table of contents for this issue, or go to the journal homepage for more

Download details:

IP Address: 90.37.249.116

The article was downloaded on 03/09/2010 at 10:29

Please note that terms and conditions apply. 


\title{
Evaluation of friction enhancement through soft polymer micro-patterns in active capsule endoscopy
}

\author{
Elisa Buselli $^{1}$, Virginia Pensabene ${ }^{2}$, Piero Castrataro $^{1}$, Pietro Valdastri ${ }^{1}$, \\ Arianna Menciassi ${ }^{1,2}$ and Paolo Dario ${ }^{1,2}$ \\ ${ }^{1}$ Scuola Superiore Sant'Anna, CRIM Lab, Piazza Martiri della Libertà 33, Pisa, Italy \\ ${ }^{2}$ Italian Institute of Technology (IIT), Center for MicroBioRobotics IIT@ SSSA, Pontedera, Italy
}

Received 13 May 2010, in final form 7 July 2010

Published 2 September 2010

Online at stacks.iop.org/MST/21/105802

\begin{abstract}
Capsule endoscopy is an emerging field in medical technology. Despite very promising innovations, some critical issues are yet to be addressed, such as the management and possible exploitation of the friction in the gastrointestinal environment in order to control capsule locomotion more actively. This paper presents the fabrication and testing of bio-inspired polymeric micro-patterns, which are arrays of cylindrical pillars fabricated via soft lithography. The aim of the work is to develop structures that enhance the grip between an artificial device and the intestinal tissue, without injuring the mucosa. In fact, the patterns are intended to be mounted on microfabricated legs of a capsule robot that is able to move actively in the gastrointestinal tract, thus improving the robot's traction ability. The effect of micro-patterned surfaces on the leg-slipping behaviour on colon walls was investigated by considering both different pillar dimensions and the influence of tissue morphology. Several in vitro tests on biological samples demonstrated that micro-patterns of pillars made from a soft polymer with an aspect ratio close to 1 enhanced friction by $41.7 \%$ with regard to flat surfaces. This work presents preliminary modelling of the friction and adhesion forces in the gastrointestinal environment and some design guidelines for endoscopic devices.
\end{abstract}

Keywords: endoscopy, friction, adhesion, soft polymer

(Some figures in this article are in colour only in the electronic version)

\section{Introduction}

This work presents the design, fabrication and testing of microfabricated polymeric structures to be integrated onto the legs of a motorized endoscopic capsule robot that is able to move in the gastrointestinal tract.

Encapsulating a video camera into a pill is a recent innovation in medical technology, which enables noninvasive visual diagnosis deep inside the intestine [1]. The images returned by wireless capsule endoscopes (WCEs) are extremely valuable from a medical standpoint. They can reveal the location and severity of lesions or bleeding, thus enabling the inspection of potential cancerous growths and permitting visual assessment of the overall health of the gastrointestinal tract. An extensive discussion of the clinical value of WCEs can be found in [2-4] and a review of WCE technology is reported in [5].

The device considered in this work adopts an internal motorized mechanism for obtaining active and teleoperated locomotion [6]. The robot is able to stop and move forward or backward inside the colon by means of small $(0.5 \mathrm{~mm}$ thick, $9.4 \mathrm{~mm}$ long) microfabricated Nitinol legs (figure 1). These legs, whose geometry is bio-inspired by insects, enable capsule locomotion and represent the interface between the device and the intestine walls $[7,8]$.

The intestine is a very challenging environment for active locomotion: the tissue is normally soft, visco-elastic and extremely compliant, and its morphology varies along the digestive tract. Moreover, the surface is slippery due to a mucus layer of about $1 \mathrm{~mm}$ that covers the intestinal mucosa, 


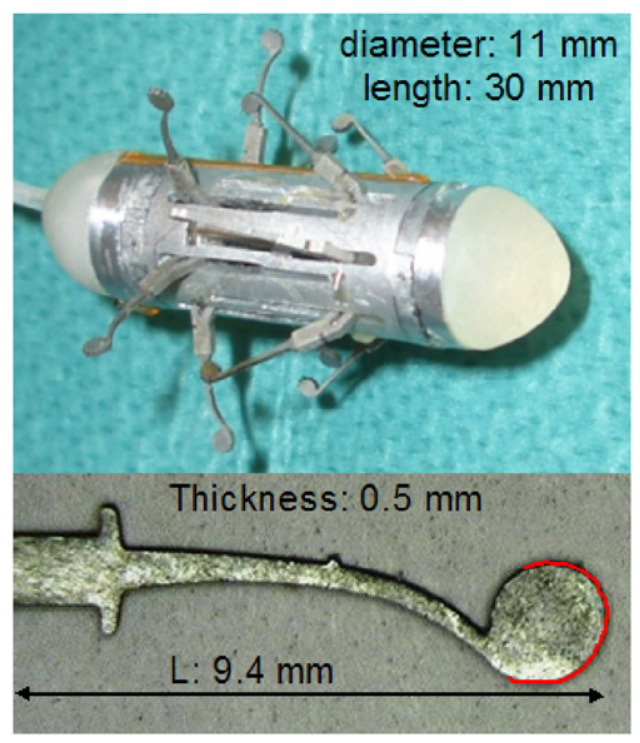

Figure 1. The endoscopic capsule and the leg. The red line highlights the contact area with the intestinal walls.

but varies in thickness and viscosity along the different tracts [9]. In this environment, the legs slip on the intestinal walls, thus impairing capsule speed and controllability. For this reason, microstructures on the leg tips made from biomimetic materials can improve their grip, thus making active locomotion more efficient. In previous works, the authors developed legs with hooks or spikes $[6,10]$, but they were not totally safe from a medical standpoint since they could cause bleeding. Thus, the main objective of this work is to find a new solution that enhances the grip between the legs and the tissue without damaging the intestinal mucosa.

Starting with the observation of nature, researchers found that several animals (e.g. tree frogs, grasshoppers and flies) have toes with a microscopically patterned structure secreting fluid in the contact area $[11,12]$. While a continuous fluid film is expected to act as a lubricant, the patterned structure enhances adhesion, thanks to its capacity to conform to rough surfaces and to prevent the propagation of cracks [13, 14]. In this context, low-aspect-ratio soft-polymer micro-pillars demonstrated enhanced adhesion when compared with flat surfaces [15].

Recently, researchers have developed synthetic structures mimicking animal adhesion [16]. However, only few studies are focused on the interaction with soft biological tissues in a wet or mucus-covered environment. In particular, in the endoscopic field, patterned capsules for the oesophagus and small intestine were presented in $[17,18]$. These works have shown how to improve the friction on the gastrointestinal tissue by covering the capsule with polymeric pillars. Another interesting study was carried out on a snail-like endoscope, which was enveloped with micro-patterned mucoadhesive film. This solution proved to be useful only in singleshot applications [19]. Different work presented a porous bioceramic material which was used to maximize the friction with intestinal walls. In particular, it was demonstrated that friction force varies with the pore size of the material [20]. An analysis of the influence of the shape of samples that slide on intestinal tissue was presented in [21], where coupons having different shapes (triangle, rectangular and cylindrical) were tested. The study showed how the shape of the surface affects friction with the tissue.

In the present work, the legs of an endoscopic capsule for colon inspection are modified by covering the contact area with polymeric microfabricated pillars. In vitro tests are performed to investigate the adhesion and friction forces between the micro-pattern and the colon tissue, thus observing the effect of different pillar dimensions on enhancing grip.

\section{Micro-pattern fabrication}

Two soft and biocompatible materials were selected for pattern fabrication: polydimethylsiloxane (PDMS, bicomponent, Sylgard 184, Dow Corning Inc.) and perfluoropolyether (PFPE, bicomponent, 5112X, Solvay Solexis). These materials are widely used in micro-patterning due to their ease of manipulation and elasticity $\left(E_{\mathrm{PDMS}} \approx 0.5-3 \mathrm{MPa}, E_{\mathrm{PFPE}} \approx 3-\right.$ $4 \mathrm{MPa}$ ). The second polymer is chosen in particular when both the fidelity of reproduction and chemical inertia are required [22].

The polymeric films were fabricated by soft lithography. The master moulds were obtained with a negative photoresist SU-8 (SU-8 50, MicroChem Corp.). It was spin-coated onto a silicon wafer with a thickness of about $100 \mu \mathrm{m}$. The thickness of the SU-8 layer determines the height of the resulting micropillars. High-resolution masks were designed and used to transfer the chosen patterns during an ultraviolet (UV) light exposure step. The moulds were maintained standing for a few minutes with trimethylchlorosilane (TMCS) before pouring the polymer, in order to facilitate the peeling-off operation.

A replica of the micro-pillar arrays was obtained by spincoating PDMS (with a monomer catalyst ratio of 10:1) at 500 rpm for $60 \mathrm{~s}$ on the SU-8 moulds. After peeling, the PDMS had the positive structure of the moulds, thus presenting a series of micro-pillars. At the same time, the PFPE samples were obtained by spin-coating the polymer ( $4 \%$ concentration) into the moulds and curing them under UV light.

Pillars having different dimensions were produced, ranging from $15 \mu \mathrm{m}$ to $180 \mu \mathrm{m}$ in diameter, with edge-toedge spacing of $75 \%$ of the pillar diameter. The maximum pillar diameter was fixed at $180 \mu \mathrm{m}$ in order to have at least two rows of pillars on the top of the leg profile. The height was fixed at $100 \mu \mathrm{m}$ for all samples [18]. A summary of sample features is given in table 1 . The final shape of the microfabricated films is a thin sheet $(100 \mu \mathrm{m}$ thick $)$ with a central micro-patterned area of $2.25 \mathrm{~cm}^{2}$ (figure 2).

At the end of the characterization phase, the micropatterns were attached to the leg, thus covering the area that contacts the intestinal tissue during capsule locomotion. The final configuration of the 'patterned leg' is shown in figure 3 . In order to facilitate bonding, a thin layer of titanium $(20 \mathrm{~nm})$ was sputtered onto the backside of the polymeric sample, which was then glued to the leg using a cyanoacrylate adhesive. 
Table 1. Micro-pattern geometry.

\begin{tabular}{lll}
\hline Pillar diameter $(\mu \mathrm{m})$ & Spacing (edge-to-edge) $\mu \mathrm{m})$ & Height $(\mu \mathrm{m})$ \\
\hline Flat surface & - & - \\
15 & 11.5 & 100 \\
60 & 45 & 100 \\
100 & 75 & 100 \\
140 & 105 & 100 \\
180 & 135 & 100 \\
\hline
\end{tabular}
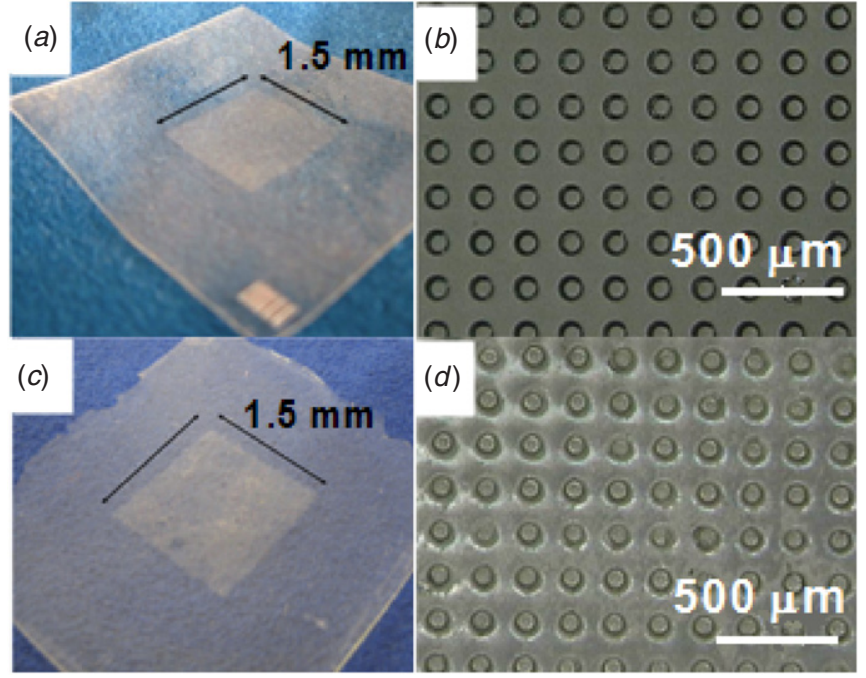

Figure 2. Patterned film and a magnification of the pillar array (100 $\mu \mathrm{m}$ in diameter) imaged by an optical microscope for PDMS $(a),(b)$ and PFPE $(c),(d)$.

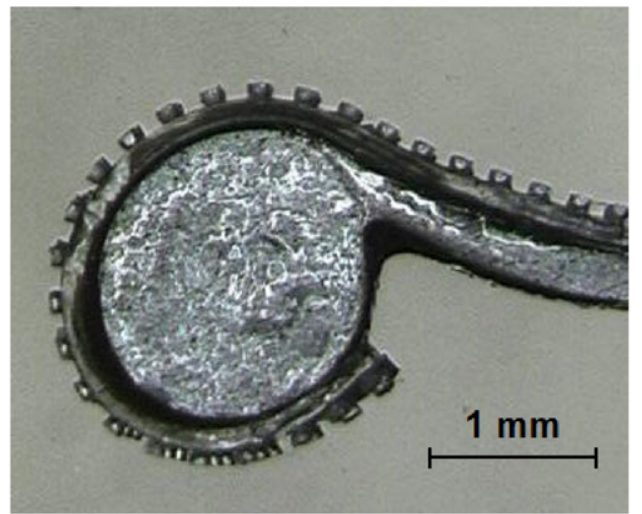

Figure 3. The leg covered with the polymeric pattern.

\section{In vitro experiments}

In vitro tests on biological samples were performed to evaluate the ability of the microfabricated samples to enhance adhesion to and friction with the tissue. In all experiments, freshly harvested porcine colon was cleaned with a physiological solution, paying attention to keep the mucus layer that covers the mucosa in in vivo conditions, which plays a key role in friction management. The experiments were conducted at room temperature. Each test was repeated eight times on each polymeric sample sliding on different specimens of colonic tissue to achieve statistical relevance, and the average values

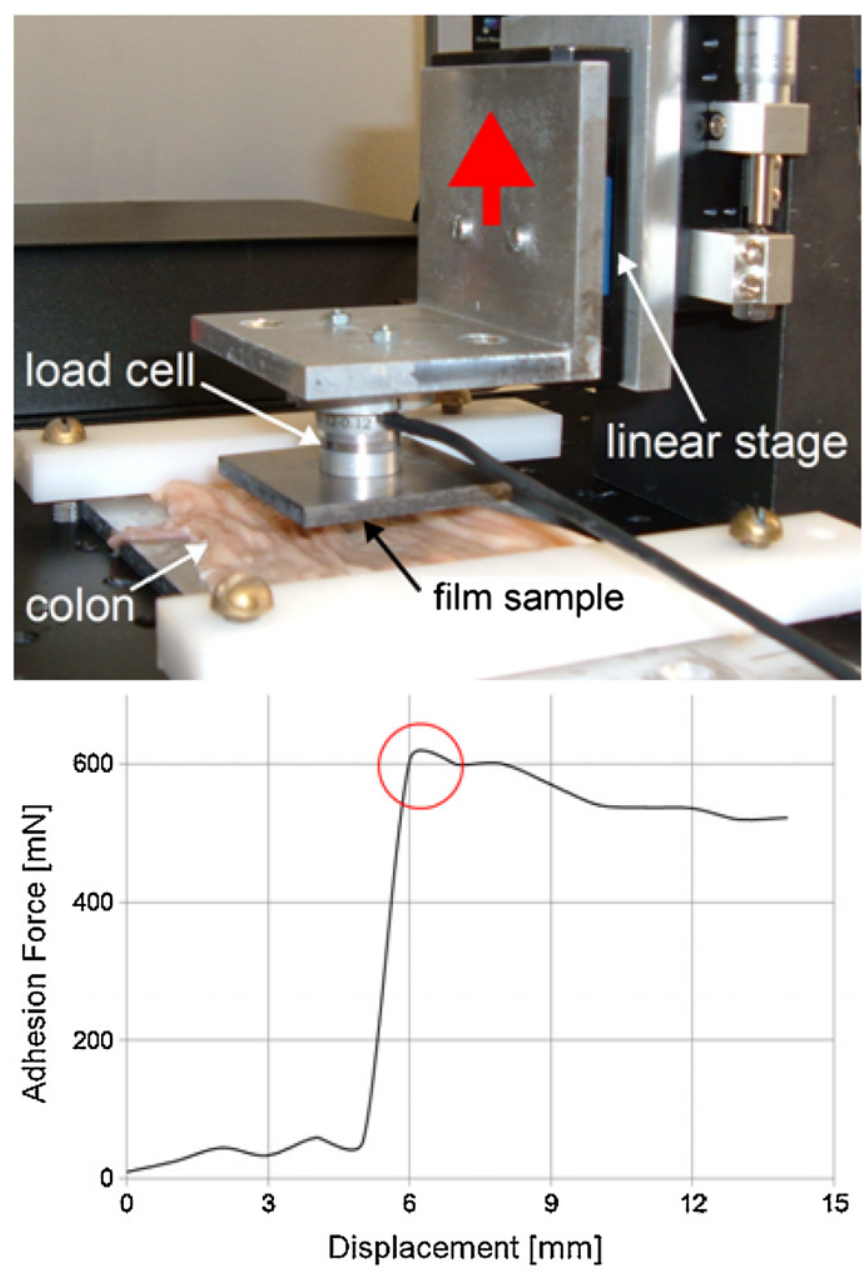

Figure 4. Adhesion experiments: set-up and typical curve.

are reported in the following part of the paper. In order to evaluate whether different polymeric materials could affect the results, the tests were carried out on both the PDMS and the PFPE samples.

After each experiment, a visual check was carried out on the tissue in order to evaluate the safety of the developed structure.

\subsection{Adhesion experiments}

For the adhesion experiments (figure 4), the micro-pattern samples were attached underneath a plate connected to a sixcomponent load cell (Nano 17, ATI, resolution 1/1280 N). The load cell was mounted on a servo-controlled linear stage (M-111.1DG, PI, Germany), fixing the speed at $0.7 \mathrm{~mm} \mathrm{~s}^{-1}$. Customized Labview software was developed to control the stage while gathering data from the load cell. The tissue was clamped to a tissue holder and the sample was maintained in contact with the tissue for $10 \mathrm{~s}$ at $10 \mathrm{mN}$ of preload before starting the lifting test. An example of a typical curve of adhesion force versus displacement obtained from this test is shown in figure 4, where the maximum peak represents the detachment force. 


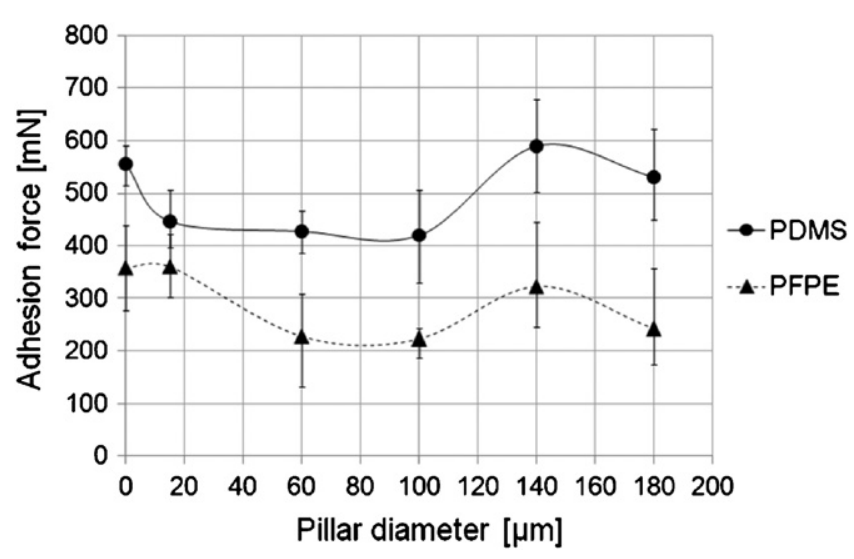

Figure 5. Graph of adhesion force versus pillar diameter.

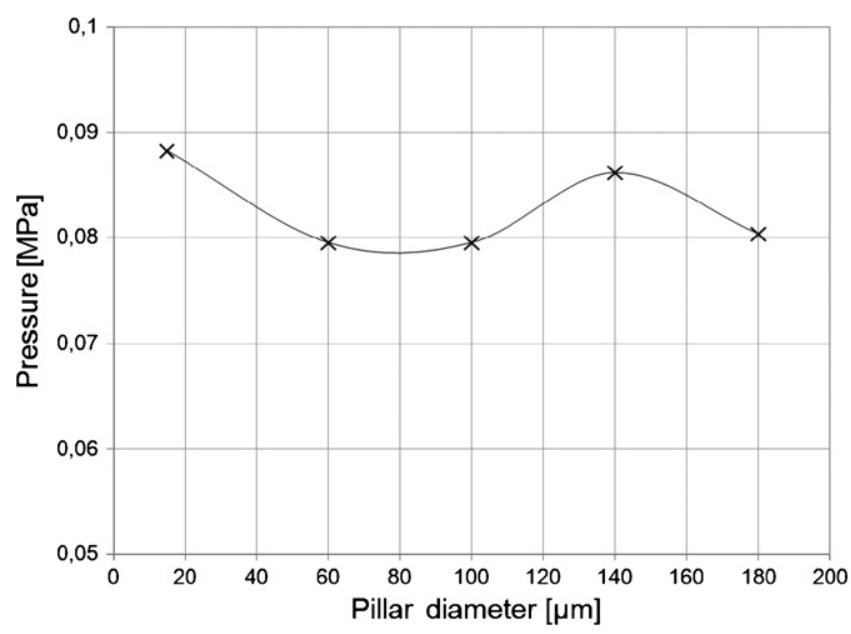

Figure 6. Graph of pressure versus pillar diameter. Pressure is estimated by dividing the normal load for the sum of the pillar top surfaces.

The acquired data allowed us to derive the experimental relation between the adhesion force and the pillar diameter for both materials (figure 5).

The two materials reveal the same trend. The flat sample exhibits large adhesion because of the wide contact area with the tissue. Under the applied preload, the soft tissue conforms to the structured sample, as already highlighted in the literature [23]. Even if this indentation is hard to measure, it can be observed that it is proportional to the pressure on the sample, estimated by dividing the normal load by the sum of the pillar top surfaces (figure 6).

The pressure exhibits a trend similar to the curve of the adhesion force. This analogy highlights that the adhesion depends on the contact pressure. The higher the pressure on the surface, the greater the indentation of the tissue, and, as a consequence, the adhesion force increases.

After the end of the test session, no visible injuries were found on the tissue.

\subsection{Friction experiments}

Friction experiments are expected to give the most significant guidelines for the pattern design, by considering that the legs generally slide on the tissue during capsule locomotion.
In the friction tests (figure 7), the samples were attached underneath a weight case, which was connected to the load cell by a thin nylon wire. The weight case had a base with round edges in order to avoid stress intensification. The applied load on the tissue was varied from $0.1 \mathrm{~N}$ up to $1 \mathrm{~N}$. This range includes the force exerted by the leg on the colonic walls, which is about $0.6 \mathrm{~N}$ [6]. The load cell was fixed on a servocontrolled linear stage (M-410.CG, PI, Germany) in order to obtain sliding of the case along the colon's longitudinal axis as the capsule moved along the gastrointestinal tract. The stage speed was $0.35 \mathrm{~mm} \mathrm{~s}^{-1}$, which is about the average speed of peristalsis (ranging from 0.16 to $0.5 \mathrm{~mm} \mathrm{~s}^{-1}$ ) [24]. It is worth mentioning that additional tests were performed by varying the slider speed from 0.2 to $0.7 \mathrm{~mm} \mathrm{~s}^{-1}$. These trials did not show a significant change in the friction force versus pillar diameter trend. An example of the typical plot of friction force versus displacement registered for one sample is shown in figure 7 , where the maximum peak represents the static friction force.

A graph of the static friction force versus the applied load was also obtained. The plot for the PDMS samples is shown in figure 8. An analogous graph was obtained for the PFPE samples.

It is clear that the static friction force increases with the applied load, as predicted by the Coulomb law $(F=$ $\mu \cdot N)$, where $N$ is the normal force and $\mu$ is the static friction coefficient. The constant $\mu$ can be derived from the slope of the linear fit of the data in the graph. By extrapolating the curve for zero preload, the lines do not pass through the origin, as predicted by the theoretical law. Thus, the expression for the total friction force can be formulated as

$$
F_{\text {Tot }}=\mu \cdot N+F_{v},
$$

where $\mu \cdot N$ (Coulomb friction) is proportional to the normal preload, and the constant $F_{v}$ represents the visco-adhesive force contribution, which is related to the physical-chemical properties of tissue and mucus. This constant contributes to the total force for values ranging between 27 and $47 \mathrm{mN}$.

The plots in figure 9 show the friction force and the friction coefficient versus the pillar diameter for both materials.

It can be observed that the micro-pattern enhances friction in comparison to the flat surface. This result is confirmed for both materials; thus, it can be ascribed to the presence of pillars, independent of material characteristics. The low friction observed for the flat sample can be associated with the mucus, which forms a continuous layer of fluid acting as a lubricant. The grooves within the patterned samples instead allow the mucus to fill the spaces between the pillars. This phenomenon can be read as a solid-to-solid contact between the pillars' top surface and tissue, which avoids hydroplaning, as reported in [25]. The maximum measured friction coefficient $(\mu=1.97)$ was achieved for the sample with $100 \mu \mathrm{m}$ pillars. This corresponds to a pillar aspect ratio of

$$
\alpha=h / d \approx 1,
$$

where $h$ is the pillar height and $d$ is the pillar diameter. Thus $\alpha$ can be used as an empirical descriptor that highlights the 

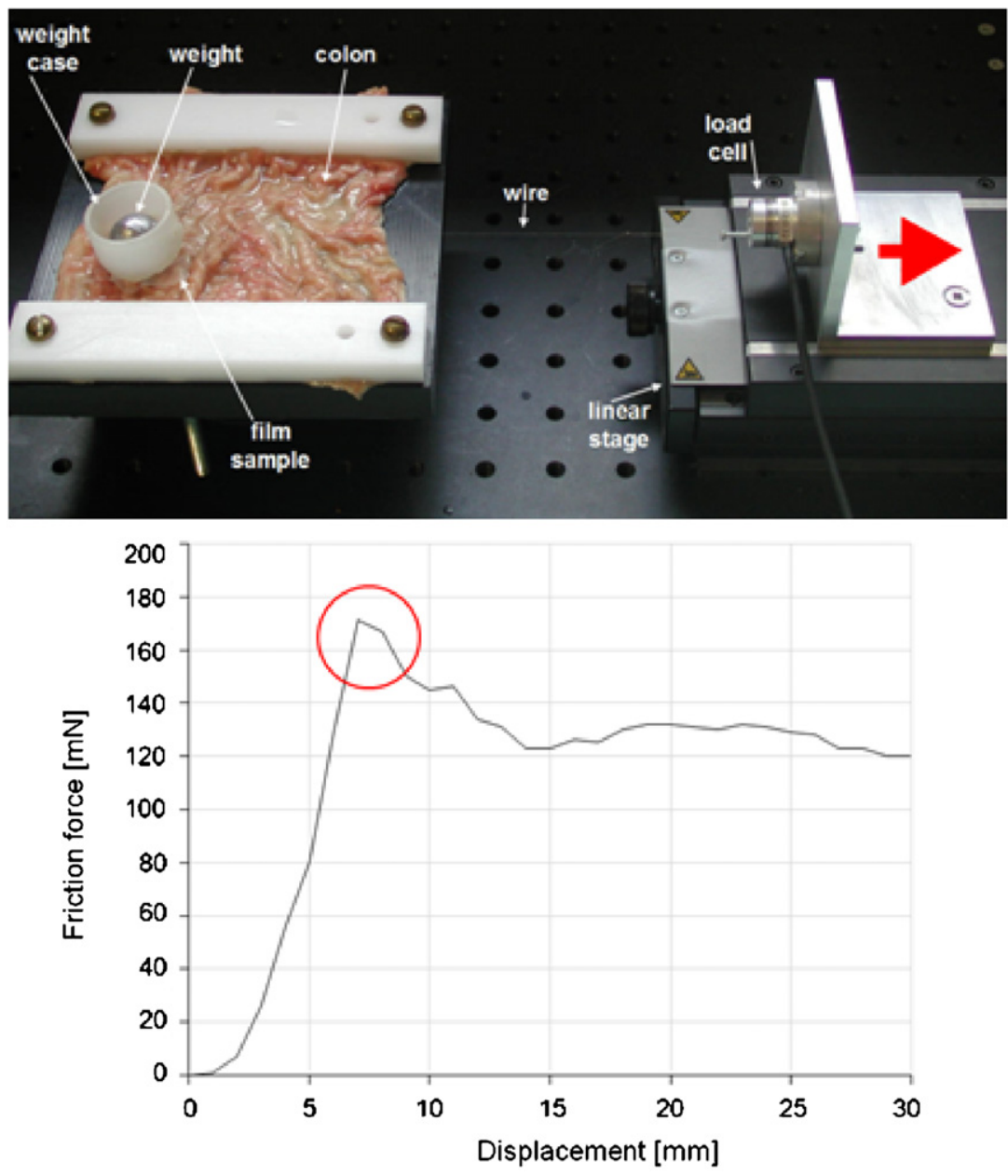

Figure 7. Friction experiments: set-up and typical curve.

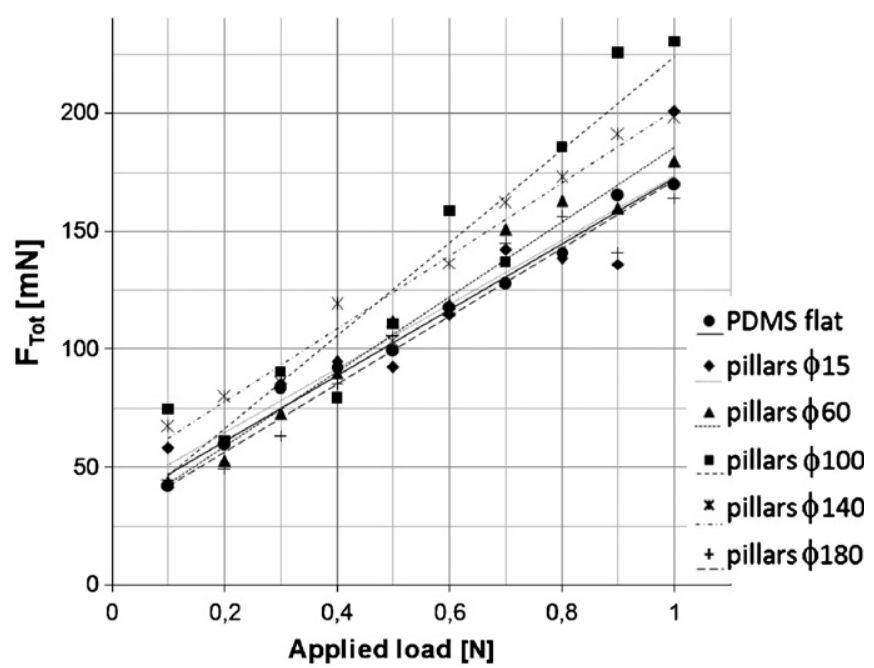

Figure 8. Graph of friction force versus applied load for PDMS samples.

ability of the patterned surface to enhance the friction with the tissue.

Working with soft biological materials $\left(E_{\text {Tissue }} \approx 1-2 \mathrm{kPa}\right.$ ) implies that the tissue conforms to the more rigid polymeric pattern when it finds enough space between the pillars [23].
The pillars with $\alpha \approx 1$ represent a sort of trade-off between the needs of maximizing the width of the grooves and the number of discontinuities in the surface unit. In fact, while the sample with low aspect ratio pillars $(\alpha<1)$ has few discontinuities (considering the same sample area), the high aspect ratio pillars $(\alpha>1)$ show very narrow grooves. Moreover, they cannot sustain high preload due to the low mechanical properties of the soft polymers, which can cause pillar bending.

At the end of the test session, no visible injuries were found on the intestinal tissue.

\subsection{Experiments on tissue morphology}

A second friction test was then conducted in order to investigate the relationship between the tissue morphology and the pattern. The colon has millimetric waviness elements in the surface topology, which are due to the inner structure and to the external shape of the intestine (figure 10).

The average profile morphology $\delta$ was measured by a laser sensor (optoNCDT 1401, Micro-Epsilon, Germany) and it was defined as the maximum peak-to-valley height,

$$
\delta=h_{\max }-h_{\min } .
$$

The test was performed with the PDMS $100 \mu \mathrm{m}$ pillar sample, which showed how to produce the highest friction, using the 

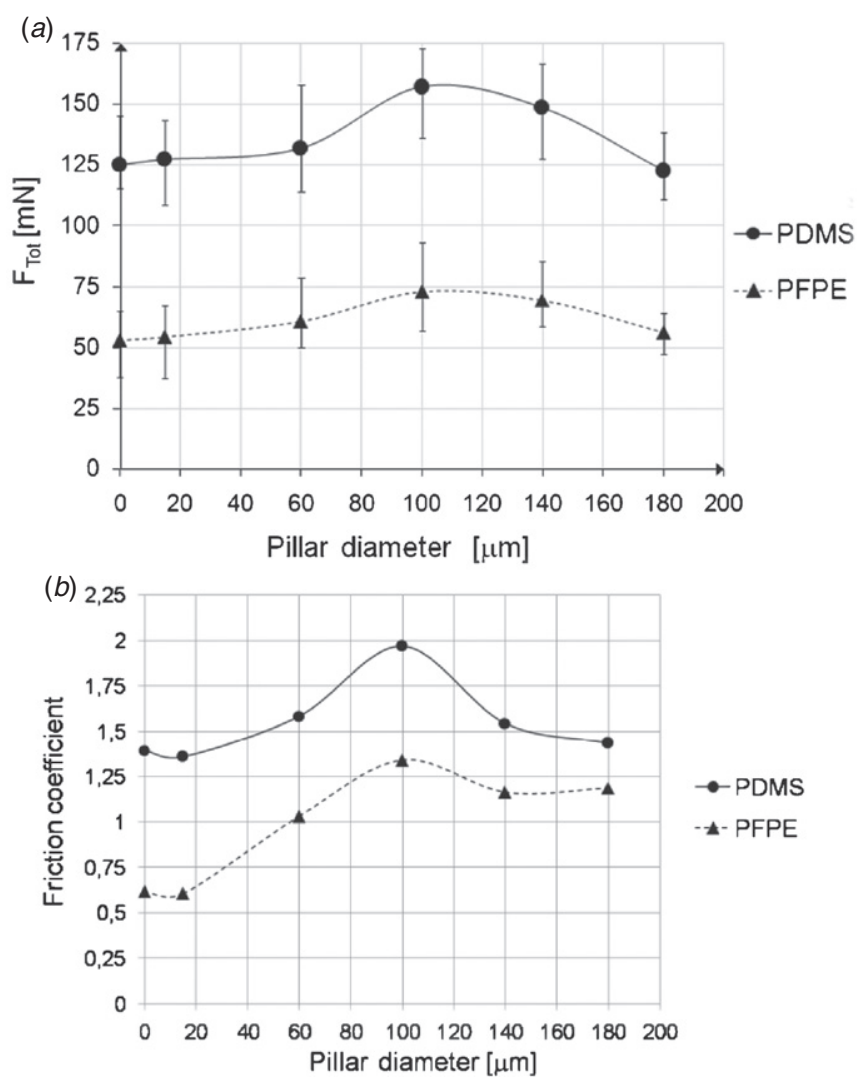

Figure 9. Friction force versus pillar diameter $(a)$ and friction coefficient versus pillar diameter $(b)$. The friction force was calculated for a load value of $0.6 \mathrm{~N}$, which is the leg pulling force on the tissue.
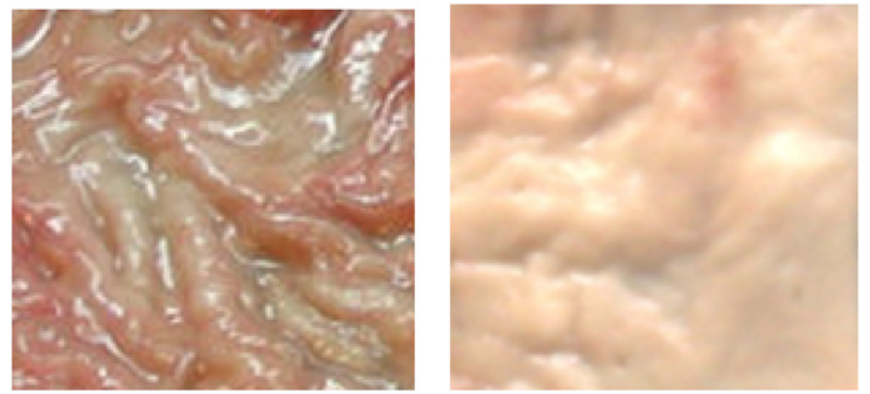

Figure 10. Colonic tissue with different profile morphologies.

same set-up as the previous test. The sample was slid on two sections of colonic tissue with different morphologies ( $\delta=1.181 \mathrm{~mm}, \delta=0.717 \mathrm{~mm}$ ) corresponding to the initial and final parts of the colon. For comparison, the test was repeated on a glass $(\delta \approx 0)$ covered with a layer of intestinal mucus.

When two sliding surfaces present comparable roughness, the friction should reach a maximum value. In fact, the intestinal profile morphology is characterized by values of $\delta$ that are noticeably bigger than the pillars $(0.7<\delta<$ $1.8 \mathrm{~mm}$ versus $h=0.1 \mathrm{~mm}$ ). On the other hand, by considering the microscopic roughness of the tissue, it would be significantly smaller (around hundreds of nanometres) than the microfabricated pillars. Thus, it is not easy to define a specific relationship between the morphology of the tissue,

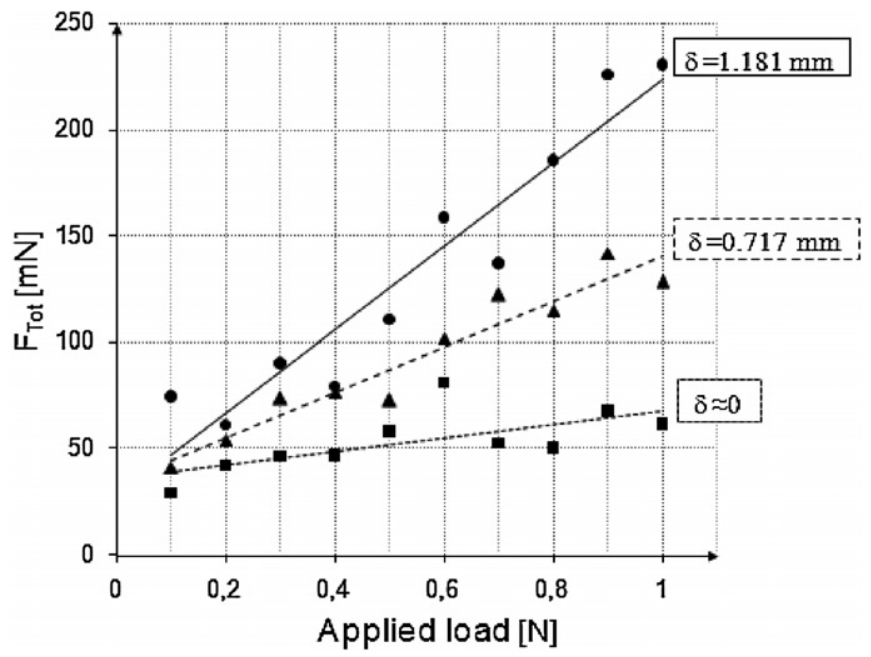

Figure 11. Graph of the friction force versus applied load for different tissue morphologies.

the pillar diameter and the friction behaviour. The tests reveal a maximum friction force on the tissue with the highest $\delta$ value (figure 11).

The most significant result is not just the numerical value but the evidence that the friction is very dependent on the local tissue morphology. This variability implies that the efficacy of patterns in a real endoscopy scenario is dependent on which part of the intestine has to be explored. Unfortunately, the morphology of the intestinal tissue of each patient cannot be predicted. However, depending on the gastrointestinal tract to be examined, the most indicated pattern can be suggested.

\section{Conclusions}

We have presented a solution to enhance the grip of an endoscopic device on the colon wall, through microfabricated patterns. Films of soft polymer structured with micro-pillars were fabricated and tested. In vitro experiments showed the influence of the patterning on the interaction with tissue. In particular, friction is related to the pillar aspect ratio. When this geometrical parameter is close to 1 , the friction coefficient undergoes an increment of $41.7 \%$. The patterned structure allows the soft intestinal tissue to conform to the polymeric surface. In addition, the mucus can fill the space in the grooves, improving the grip. This empirical rule can be used as a guideline for further work in the field of endoscopic devices.

The adhesion and friction tests gave different values of optimum diameters. It is worth mentioning that they are different phenomena, thus there is no evidence a priori supporting the same value. In our case, we put more emphasis on the friction tests (choosing $100 \mu \mathrm{m}$ as the preferable diameter of the pillars) because the movement of the leg resembles more a sliding than an adhesion gait.

A visual check on all tissues after the experiments revealed no injures to the mucosa. This confirmed that the developed structure is completely safe and compatible for the application of a medical device.

Nowadays, despite the broad literature, there is a lack of reliable data on tissue morphology and mucus properties, 
which could in future help to optimize the structure. Future work will be dedicated to evaluating the optimal pillar height as tissue features change in different areas of the intestinal tract. In addition, in vivo experiments will be carried out in a real colonoscopy to verify the improvement in the endoscopic capsule speed and controllability.

\section{Acknowledgments}

This work is supported in part by the Intelligent Microsystem Center, KIST, South Korea, in the framework of the OPTIMUS Project (optimization and validation of a mobile capsule for endoscopy), and in part by the European Commission in the framework of VECTOR FP6 European Project (versatile endoscopic capsule for gastrointestinal tumor recognition and therapy). The authors are also grateful to R Sahai and C Filippeschi for their technical support.

\section{References}

[1] Iddan G, Meron G, Glukhovsky A and Swain P 2000 Wireless capsule endoscopy Nature $\mathbf{4 0 5} 417$

[2] Waterman M and Eliakim R 2009 Capsule enteroscopy of the small intestine Abdom. Imaging 34 452-8

[3] Van Gossum A et al 2009 Capsule endoscopy versus colonoscopy for the detection of polyps and cancer N. Engl. J. Med. $\mathbf{3 6 1} 1220$

[4] Karargyris A and Bourbakis N 2010 Wireless capsule endoscopy and endoscopic imaging IEEE Eng. Med. Biol. Mog. 29 72-83

[5] Moglia A, Menciassi A, Schurr M and Dario P 2007 Wireless capsule endoscopy: from diagnostic devices to multipurpose robotic systems Biomed. Microdevices $9235-43$

[6] Valdastri P, Webster R J III, Quaglia C, Quirini M, Menciassi A and Dario P 2009 A new mechanism for meso-scale legged locomotion in compliant tubular environments IEEE Trans. Robot 25 1047-57

[7] Quirini M, Menciassi A, Stefanini C, Gorini S, Pernorio G and Dario P 2005 Development of a legged capsule for the gastrointestinal tract: an experimental set-up Proc. IEEE ROBIO (Hong Kong) pp 161-67

[8] Buselli E, Valdastri P, Quirini M, Menciassi A and Dario P 2009 Superelastic leg design optimization for an endoscopic capsule with active locomotion Smart Mater. Struct. 18015001

[9] Atuma C, Strugala V, Allen A and Holm L 2001 The adherent gastrointestinal mucus gel layer: thickness and physical state in vivo Am. J. Physiol. Gastrointest. Liver Physiol. 280 G922-29

[10] La Spina G, Stefanini C, Menciassi A and Dario P 2005 A novel technological process for fabricating micro-tips for biomimetic adhesion J. Micromech. Microeng. 15 1576-87

[11] Scherge M and Gorb S N 2001 Biological Micro- and Nanotribology: Nature's Solutions (Berlin: Springer))

[12] Federle W, Barnes W J P, Baumgartner W, Drechsler P and Smith J M 2006 Wet but not slippery: boundary friction in tree frog adhesive toe pads $J$. R. Soc. Interface 3 689-97

[13] Hui C Y, Glassmaker N J, Tang T and Jagota A 2004 Design of biomimetic fibrillar interfaces: 2 mechanics of enhanced adhesion J. R. Soc. Interface $135-48$

[14] Chung J Y and Chaudhury M K 2005 Roles of discontinuities in bio-inspired adhesive pads J. R. Soc. Interface 2 55-61

[15] Crosby A J, Hageman M and Duncan A 2005 Controlling polymer adhesion with "pancakes" Langmuir 21 11738-43

[16] Gorb S N, Sinha M, Peressadko A, Daltorio K A and Quinn R D 2007 Insects did it first: a micropatterned adhesive tape for robotic applications Bionsp. Biomim. 2 S117-25

[17] Glass P, Cheung E, Wang H, Appasamy R and Sitti M 2008 A motorized anchoring mechanism for a tethered capsule robot using fibrillar adhesives for interventions in the esophagus Proc. 2nd IEEE/RAS BIOROB (Scottsdale) pp 19-22

[18] Kwon J, Cheung E, Park S and Sitti M 2006 Friction enhancement via micro-patterned wet elastomer adhesives on small intestinal surfaces Biomed. Mater. 1216-20

[19] Dodou D, del Campo A and Artz E 2007 Mucoadhesive micropatterns for enhanced grip Proc 29th IEEE EMBS (Lyon) pp 1457-62

[20] Wang X, Han Y, Jiang X, Dai H and Li S 2006 Static friction of porous bioceramic $\beta$-TCP on intestinal mucus film Biomed. Mater. 1 124-6

[21] Wang K D and Yan G Z 2009 Research on measurement and modelling of the gastro intestine's frictional characteristics Meas. Sci. Technol. 20015803

[22] Rolland J P, Van Dam R M, Schorzman D A, Quake S Q and DeSimone J M 2004 Solvent-resistant photocurable "liquid Teflon" for microfluidic device fabrication $J$. Am. Chem. Soc. 126 2322-3

[23] Mahdavi A et al 2008 A biodegradable and biocompatible gecko-inspired tissue adhesive Proc. Natl Acad. Sci. 105 2307-12

[24] Martini F H 1995 Fundamentals of Anatomy \& Physiology (Englewood Cliffs, NJ: Prentice-Hall)

[25] Varenberg M and Gorb S N 2009 Hexagonal surface micropattern for dry and wet friction Adv. Mater. 21 483-6 